\section{Elisabeth Chroni \\ Andreas A. Argyriou \\ Panagiotis Polychronopoulos \\ Vassiliki Sirrou}

\title{
The effect of stimulation technique on sympathetic skin responses in healthy subjects
}

Abstract The aim of this study was to collect normative data for sympathetic skin responses (SSR) elicited by electrical stimulus of the ipsilateral and contralateral peripheral nerves, and by magnetic stimulus of cervical cord. SSRs were measured at the midpalm of both hands following electrical stimulation of the left median nerve at the wrist and magnetic stimulation at the neck in 40 healthy adult volunteers (mean age $52.2 \pm 12.2$ years, 19 males). The onset latency, peak latency, amplitude and area were estimated in "P" type responses (i.e., waveforms with a larger positive, compared to negative, component). SSR onset and peak latency were prolonged when the electrical stimulus was applied at the contralateral side (i.e., the SSR recorded in the right palm $P<0.001)$. The onset latency was similar on both sides during cer- vical magnetic stimulation. However, peak latency was faster on the left side $(P<0.03)$. Comparison of electrical and magnetic stimulation revealed that both the onset and peak latency were shorter with magnetic stimulation $(P<0.001)$. The latency of a SSR varies depending on what type of stimulation is used and where the stimulus is applied. Electrically generated SSRs have a longer delay and the delay is prolonged at the contralateral side. These factors should be taken into account when interpreting SSR data.

Key words sympathetic skin response galvanic skin response sudomotor function . methods $\cdot$ autonomic nervous system

\section{Introduction}

Sympathetic skin response (SSR) testing is commonly used to assess sympathetic function [18]. Abnormal SSR occur in both central $[7,8]$ and peripheral autonomic dysfunction $[1,16,21]$.

The SSR is a complex polysynaptic reflex, which courses through a common centrifugal path via J sympathetic post-ganglionic unmyelinated fibers [2,
24]. However, its exact pathway through the central nervous system is not fully understood. A variety of stimuli can generate a SSR, which illustrates the complexity of the afferent inputs to the reflex.

Although measuring SSR is relatively simple, interpreting these measurements is not so straightforward. Different afferent stimuli may well lead to a wide range of normal SSR values. It is difficult to attribute abnormalities of SSR to sympathetic dysfunction alone, particularly in cases of polyneuropa- 
thy, due to the potential influences by somatic fibers sensory inputs [24].

The aim of this study was to collect normative SSR data. SSRs were measured in both mid-palms following electrical stimulation of the left medial nerve and magnetic stimulation of the neck in healthy controls. SSR onset and peak latency, amplitude and area were analyzed. Electrically generated SSR in the ipsilateral (left) and contralateral (right) palms were compared to the SSR evoked by magnetic stimulation.

\section{Methods}

\section{Subjects}

Forty healthy adult volunteers $[9$ males/21 females, mean age $52.21 \pm 12.23$ (range 28-75) years, height $172 \pm 11.3 \mathrm{~cm}$ ] were studied. All subjects were healthy, with no history of cardiovascular or neurological disease. All subjects denied previous alcohol or drug abuse. None of the subjects had contraindications for magnetic stimulation, such as metal prosthesis or pacemaker. All participants gave written informed consent prior to the start of the study.

\section{Procedure}

The study was performed using a 2-channel Counterpoint, Medtronic-Dantec (Medtronic-Dantec Electronics, Skovlunde, Denmark) electromyographic unit. SSR recordings were based on the technique described by Shahani et al. [20]. Briefly, subjects lay supine in an air-conditioned, quiet room with temperature maintained at $24^{\circ} \mathrm{C}$ and skin temperature at $33 \pm 0.5^{\circ} \mathrm{C}$. The same investigator performed all experiments, and care was taken to avoid all external stimulation. Active standard disk ECG Ag/AG/Cl electrodes were attached to the mid-palm of both hands and referenced to the corresponding dorsal surfaces. Skin temperature was verified as being above $32^{\circ} \mathrm{C}$ by measuring close to the recording electrodes. The recording sites were the same in all experiments.

\section{Electrical stimulation}

Single square-wave electrical pulses of 0.1 milliseconds duration were applied to the left median nerve at the wrist at irregular intervals (30-60 seconds) to produce a SSR. The sweep speed was 1,000 milliseconds/D, the amplifiers sensitivity was $100 \mu \mathrm{V} /$ div and the amplifier filters were set at $0.5 \mathrm{~Hz}$ and $2 \mathrm{kHz}$ for the low and high frequency filter, respectively. Five trials were recorded simultaneously from both palms and on each side the response with the largest amplitude and shortest latency were taken. A fixed stimulus intensity of $60 \mathrm{~mA}$ was used throughout.

\section{Magnetic stimulation}

A Magstim 200 stimulator, equipped with a circular coil of $9 \mathrm{~cm}$ inner diameter and a 2.0-T maximum magnetic field strength, was used to apply magnetic stimulation at the cervical area. The center of the circular coil was placed in the midline over the 7th cervical spinous process. The current was set to anti-clockwise flow in the coil (side A visible) and the stimulus intensity fixed at $60 \%$ of maximum output. Five stimuli were delivered randomly every 20 30 seconds.

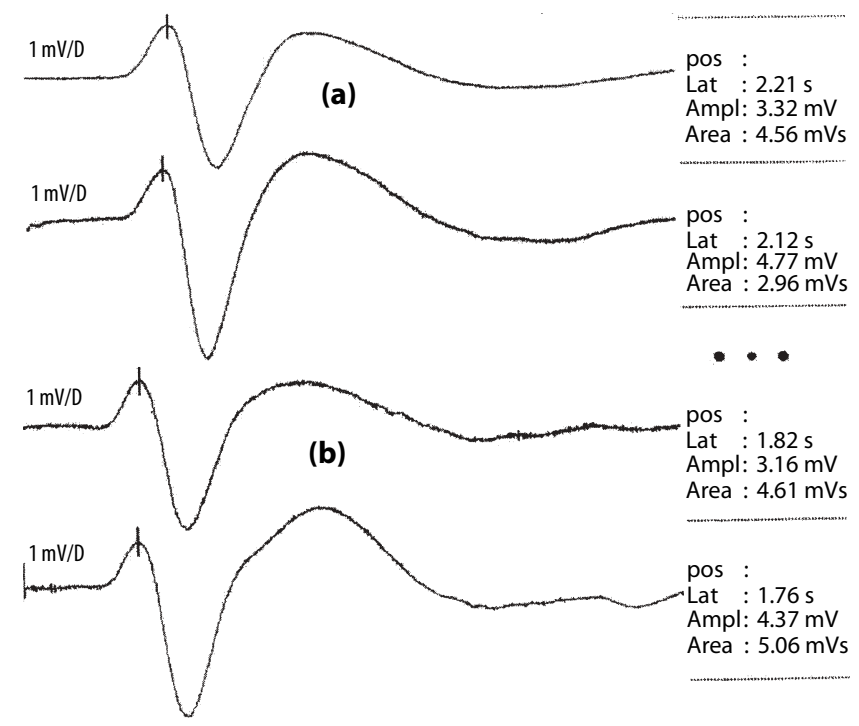

Figure 1 A typical sympathetic skin response recording. Typical SSR recordings taken from the right palm (upper trace) and left palm (lower trace) (a) following electrical stimulus of left median nerve and (b) magnetic stimulation of cervical cord

\section{Analysis of waveforms}

To avoid habituation 5 minutes rest was given between electrical and magnetic stimulation. To maintain uniformity of the results only "P" type responses (waveforms with a larger positive, compared to negative, component) were analyzed.

Figure 1 shows how the components of the waveform were analyzed. The onset latency was taken from the time of the stimulus artefact to the start of the positive deflection. The peak latency was taken from the time of the stimulus artefact to the peak of the positive deflection. The peak-to-peak amplitude and the area under the SSR curve were also measured.

\section{Statistical analysis}

Differences between stimulus procedures in the same subjects were determined by using paired $t$-tests. The tests were two tailed. Statistical significance was set at $P<0.05$. All analyses were performed using SPSS for Windows (10.0; SPSS Inc., Chicago, IL).

\section{Results}

Clear SSR recordings with a main positive component (i.e., $\mathrm{P}$ waveforms) were obtained in all participants.

\section{Latency}

Mean SSR onset and peak latency on the right side, which was contralateral to the electrical stimulus, was significantly prolonged compared to the left side, which was ipsilateral to the electrical stimuli $(P<0.001$, Tables 1 and 2$)$. No differences in the onset latency were found between right and left sides 
Table 1 Normative sympathetic skin response data in healthy subjects

\begin{tabular}{lllll}
\hline & Electrical ipsilateral (L) & Electrical contralateral (R) & Magnetic (L) & Magnetic (R) \\
\hline Latency-onset (seconds) & $1.79 \pm 0.22$ & $1.90 \pm 0.24^{\mathrm{a}}$ & $1.63 \pm 0.25^{\mathrm{c}}$ & $1.66 \pm 0.28^{\mathrm{d}}$ \\
Latency-peak (seconds) & $2.54 \pm 0.33$ & $2.68 \pm 0.38^{\mathrm{a}}$ & $2.40 \pm 0.27^{\mathrm{c}}$ & $2.43 \pm 0.28^{\mathrm{b}, \mathrm{d}}$ \\
Amplitude (mV) & $2.67 \pm 1.49$ & $2.35 \pm 1.32^{\mathrm{a}}$ & $2.74 \pm 1.61$ & $2.70 \pm 1.69$ \\
Area (mV seconds) & $2.85 \pm 1.53$ & $2.63 \pm 1.41$ & $3.24 \pm 1.99$ & $3.22 \pm 1.96^{\mathrm{d}}$ \\
\hline
\end{tabular}

All data are mean \pm SD. Electrical stimulation was applied at the left medial nerve of the wrist. SSR were recorded in both the left (ipsilateral) and right (contralateral) palms. Statistically significant difference $(P<0.05)$

${ }^{a}$ Electrical stimulus-between sides

${ }^{\mathrm{b}}$ Magnetic stimulus-between sides

' Left side-between electrical and magnetic stimulus

${ }^{d}$ Right side- between electrical and magnetic stimulus following cervical magnetic stimulation. However, a weak interside difference in peak latency $(P<0.03)$ was observed (faster on the left side). Comparison between electrical and magnetic stimulus revealed that both onset and peak latency on either side were significantly shorter for magnetic stimulation $(P<0.001$, Tables 1 and 2$)$.

\section{Amplitude and area}

During electrical stimulation amplitude was higher of the stimulated (left) side compared to the non-stimulated side. Amplitude was similar during magnetic and contralateral electrical stimulation. The area of the SSR measured at the right side was greater for magnetic stimulation compared to electrical stimulation.

\section{Discussion}

The main finding of this study was that the latency of a SSR depends on whether an electrical or magnetic evoking stimulus is used. We also found a mean latency difference between contralateral and ipsilateral evoked electrical responses of 105 milliseconds.

SSR are generated through a complex somatosympathetic reflex with spinal, bulbar and suprabulbar components [13]. Until now, they have not been precisely defined in humans [26]. SSR can be evoked

Table 2 The difference in sympathetic skin response onset latencies evoked by electrical and magnetic stimulation

\begin{tabular}{lc}
\hline & Latency difference (milliseconds) \\
\hline Electrical stimulation. R-L & $105 \pm 85(0-320)^{*}$ \\
Magnetic stimulation. R-L & $27 \pm 86(-110-330)$ \\
Left electrical-magnetic & $158 \pm 183(-50-640)^{*}$ \\
Right electrical-magnetic & $237 \pm 201(0-780)^{*}$
\end{tabular}

Differences in SSR onset latency measurements during 3 types of stimulation (left and right electrical stimulation and magnetic stimulation). All data are mean \pm SD (and range). L, left; $\mathrm{R}$, right. * Significance of $P<0.05$ (comparison by one sample $t$-test) by various types of stimuli including auditory, deep breathing, painful sensation of the limbs or face etc., and are therefore mediated by different afferent pathways [2]. Moreover, SSR are evoked by impulses that originate in a different area to which the response is produced. For example, electrical pulses over the sternal skin can be used to elicit SSR in the palms [19], which highlight the complexity of the internal neuronal network involving polysynaptic relays and connections between different vertical levels along the neuraxis and in both sides of the human body [11]. It is, therefore, not surprising that measurement of a SSR depends on the site and the type of stimulus used to initiate the reflex.

The longer latency in the contralateral side of 105 milliseconds may be explained by the crossing over of the signal from one side of the body to the other. The cross over time of around 100 milliseconds suggests that the reflex circuits are supraspinal [13]. Data from animal models indicate that the crossing occurs at multiple levels, including the brainstem and spinal cord [26]. However, patients with cervical or high thoracic lesions resulting in complete tetraplegia or paraplegia have no SSR from the hands and feet [6], suggesting that supraspinal centers are essential in the generation of SSR.

A constant latency to alternatively electrical stimulation of median nerves was suggested in a previous study [3]. However, latency was measured with 20 consecutive electrical stimulations, and thus the effects of adaptation (or habituation) cannot be excluded.

Magnetic stimulation may be most reproducible method to evoke SSR [14]. In theory, strong stimulation generated by the magnetic field close to the spine avoids any possible interference by affected somatic sensory fibers, providing a measure of efferent sympathetic function alone. In the case of the lower motor neurons, it has been showed that cervical magnetic stimulation excites the motor roots at or very near the exit foramen or even further distally [5]. In respect of SSRs, magnetic stimulation could, in analogy to motor neurons, excite the sensory roots at 
their entry [14] or directly activate the sympathetic trunks within the spinal canal [25].

In our study, the latency of the SSR evoked by magnetic stimulation was shorter than the corresponding values of the electrically elicited responses. The shorter latency of the magnetically generated SSR could be caused by impulses bypassing the peripheral afferent part of the reflex. Given that the difference between electrical stimulation at the wrist and magnetic stimulation at the neck was 158 milliseconds and assuming that the average distance from wrist to C7 is $70 \mathrm{~cm}$, the conduction velocity of afferent somatic fibers is calculated to be approximately $5 \mathrm{~m} /$ second.

Previous studies suggested that the efferent path is served by sympathetic B and C fibers conducting at approximately $2 \mathrm{~m} /$ second $[12,17]$, whereas the afferent path is served by somatic myelinated sensory groups II and III conducting at rates of $50-5 \mathrm{~m} / \mathrm{sec}-$ ond [12]. In good agreement with our results, other investigators have reported a latency difference between electrically and magnetically elicited SSR of 80 milliseconds [14] and 130 milliseconds [4], with the former being always longer.

Based on our results, it can be assumed that the SSR onset latency to spinal cord magnetic stimulation was the shortest, whereas that to contralateral peripheral nerve electrical stimulation was the longest. Furthermore, the difference in latency between SSR to magnetic and ipsilateral electrical stimuli was close to that between SSR to ipsilateral and contralateral electrical stimuli. A satisfactory explanation for the observed differences would not be offered without the risk of being highly speculative.

The morphology is another element of SSRs instability. Two main types of SSR waveform were previously recognized; $P$ type which has a larger positive component and $\mathrm{N}$ type with a larger negative component $[15,22,23]$. It has been shown that strength of stimulation was positively correlated with $\mathrm{P}$ type amplitude and inconsistently with $\mathrm{N}$ type and that strong stimuli probably produced $\mathrm{P}$ type responses [22]. To ensure similarity of measurements and facilitate comparison of responses to different kinds of stimuli, we arbitrarily choose to study only subjects with predominant $\mathrm{P}$ type responses. In none of our participants the change of stimulation from electrical (ipsilateral and contralateral) to magnetic resulted in switching of $\mathrm{P}$ to $\mathrm{N}$ type of SSR. This can be the consequence of using relatively high intensity in electrical and magnetic stimulus.

The latency values in our subjects were slightly higher than those in previous reports. In those reports, the mean SSR latency from the palm to electrical stimulation of the median nerve ranged between 1.27 seconds and 1.51 seconds $[4,10,14,24]$. This discrepancy should be attributed to a methodological difference. The negative onset of the response is generated by the electrical activation of the sweat gland. The peak of the negative phase is probably variable and generated by the relationship between sweat production and the surround tissue [24]. Under the particular recording conditions applied, the majority of the examined individuals had potentials with an initial positive peak. Therefore, instead of measuring the onset latency to the first negative peak, which is most often used, we measured the onset latency from stimulus artifact to the onset of the main positive wave irrespective of the existence or not of a proceeding small negative component. As regards to the peak latency values, they showed similar to onset latency differences in this study. Thus, peak latency is not suggested as an additional parameter, which could be useful in clinical practice.

Amplitude is the SSR parameter that has been reported to be highly variable. It can be influenced by several factors such as temperature, time of the day, emotion and for this reason several authors have not used it as measure of disease $[2,9]$. The relative stability of amplitude and area measurements among the SSRs evoked by different stimuli in our subjects is noticeable. These findings could be attributed to the stable conditions of recordings (single session for all SSR recordings in each subject and long interstimulus interval to avoid habituation).

There are certain potential limitations in the study design. First, the fact that SSRs were not assessed for reproducibility in individual subjects. However, the phenomenon of habituation [3] did not allow us to compare measurements (particularly for the amplitude) of consecutive responses in a single individual.

Second, since it is known that a variety of stimuli are capable of producing SSR, startle could intermix with the electrical stimulus and, mostly, auditory could interfere with the magnetic stimulus, leading to shorter SSR latencies. A study comparing parameters of SSR elicited by pure auditory as opposed to other types of stimulation should clarify this point. However, in a previous study, where ear-plugs were used to exclude auditory stimuli, the SSR latency measurements to neck magnetic stimulation were even shorter than in our subjects [14], suggesting that the intermixed auditory stimulation is not a major limitation. Finally, habituation, in a broad sense including the central processing and familiarity, cannot be completely ruled out by a 5 -minute interval between techniques, although intervals of 40-60 seconds between trials were reported elsewhere [10, 14, 22]. Application of electrical followed by magnetic stimulation in some subjects and the inverse procedure in others might have been used, although this would have increased the complexity of the study design. 
In brief, SSRs' have been introduced as an easy to perform and painless mean for the evaluation of postganglionic sympathetic function [20]. In the filed of autonomic nervous system where the diagnostic options are limited, and despite the expression of certain doubts, mainly related to the large diversion of individual values, SSR remains at present the most widely applied neurophysiological test for the sympathetic nervous system. According to the results of the present study, latency which is the most widely quoted parameter, varied depending on the stimulation type, being shorter at ipsilateral than contralateral electrical stimulus and even less short when magnetic stimulation at the neck was applied. Further studies on the challenging issue of SSR waveform and its possible clinical relevance are necessary to explore it in detail.

\section{References}

1. Argyriou AA, Koutras A, Polychronopoulos P, et al. (2005) The impact of paclitaxel or cisplatin-based chemotherapy on sympathetic skin response: a prospective study. Eur J Neurol 12(11):858-861

2. Arunodaya GR, Taly AB (1995) Sympathetic skin response: a decade later. J Neurol Sci 129:81-89

3. Cariga P, Catley M, Mathias CJ, Ellaway PH (2001) Characteristics of habituation of the sympathetic skin response to repeated electrical stimuli in man. Clin Neurophysiol 112:1875-1880

4. Chu EC, Chu NS (1997) Patterns of sympathetic skin response in palmar hyperhidrosis. Clin Aut Res 7:1-4

5. Cros D, Chiappa KH, Gominak S, Fang J, Santamaria J, King PJ, Shahani BT (1990) Cervical magnetic stimulation. Neurology 40:1751-1756

6. Curt A, Weinhardt C, Dietz V (1996) Significance of sympathetic skin response in the assessment of autonomic failure in patients with spinal cord injury. J Auton Nerv Syst 61:175-180

7. Dettmers C, Fatepour D, Faust H, Jerusalem F (1993) Sympathetic skin response abnormalities in amyotrophic lateral sclerosis. Muscle Nerve 16(9):930-934

8. Drory VE, Nisipeanu PF, Kroczyn AD (1995) Tests of autonomic dysfunction in patients with multiple sclerosis. Acta Neurol Scand 92(5):356-360

9. Fagius J, Wallin BG (1980) Sympathetic skin latencies and conduction velocities in patients with polyneuropathy. J Neurol Sci 47:449-461
10. Haapaniemi TH, Korpelainen JT, Tolonen U, et al. (2000) Suppressed sympathetic skin response in Parkinson disease. Clin Autonom Res 10:337-342

11. Jänig W, Häbler H-J (2003) Neurophysiological analysis of target-related sympathetic pathways - from animal to human: similarities and differences. Acta Physiol Scand 177:255-274

12. Low PA (2004) Evaluation of sudomotor function. Clin Neurophysiol 115:1506-1513

13. Manca D, Valls-Solé J, Callejas MA (2000) Excitability recovery curve of the sympathetic skin response in healthy volunteers and patients with palmar hyperhidrosis. Clin Neurophysiol 11:1767-1770

14. Matsunaga K, Uozumi T, Tsuji S, Murai Y (1995) Sympathetic skin responses evoked by magnetic stimulation of the neck. J Neurol Sci 128:188-194

15. Mitani H, Ishiyama $Y$, Hashimoto I (2003) Equivalent current dipole estimated from SSR potential distribution over the human hand. Clin Neurophysiol 114:233-238

16. Nazhel B, Yetkin I, Irkec C, Kocer B (2002) Sympathetic skin response in diabetic neuropathy. Electromyogr Clin Neurophysiol 42(3):181-185

17. Parisi L, Rossi P, Valente G, Fattapposta F, Mellina V, Martini A, et al. (2001) Estimation of the conduction velocity of sympathetic sudomotor C fibres in healthy subjects: study of sympathetic skin reflex. Funct Neurol $16: 231-237$
18. Schondorf R (1993) New investigations of autonomic nervous system function. J Clin Neurophysiol 10(1):28-38

19. Sener HO, Tascilar NF, Balaban H, Selcuki D (2000) Sympathetic skin response in carpal tunnel syndrome. Clin Neurophysiol 111(8):1395-1399

20. Shahani BT, Halperin JJ, Boulu P, Cohen J (1984) Sympathetic skin response-a method of assessing unmyelinated axon dysfunction in peripheral neuropathies. J Neurol Neurosurg Psychiatry 47(5):536-542

21. Taly AB, Arunodaya GR, Rao S (1995) Sympathetic skin response in GuillainBarre syndrome. Clin Auton Res 5(4):215-219

22. Toyokura M (2003) Influence of stimulus intensity on waveform of sympathetic skin response evoked by magnetic stimulation. Clin Neurophysiol 114:1423-1430

23. Toyokura M (2006) Sympathetic skin responses: the influence of electrical stimulus intensity and habituation on the waveform. Clin Auton Res 16:130135

24. Uncini A, Pullman SL, Lovelace RE, Gambi D (1988) The sympathetic skin response: normal values, elucidation of afferent components and application limits. J Neurol Sci 87:299-306

25. Uozumi T, Nakano S, Matsunaga K, Tsuji S, Murai Y (1993) Sudomotor potential evoked by magnetic stimulation of the neck. Neurology 43:13971400

26. Vetrugno R, Liguori R, Cortelli P, Montagna P (2003) Sympathetic skin response. Clin Auton Res 13:256-270 